\title{
Strongly Correlated Electrons on a Silicon Surface: Theory of a Mott Insulator
}

\author{
C. Stephen Hellbergt and Steven C. Erwint \\ Complex Systems Theory Branch, Naval Research Laboratory, Washington, D.C. 20375
}

(November 7, 2018)

\begin{abstract}
We demonstrate theoretically that the electronic ground state of the potassium-covered $\operatorname{Si}(111)$ B surface is a Mott insulator, explicitly contradicting band theory but in good agreement with recent experiments. We determine the physical structure by standard density-functional methods, and obtain the electronic ground state by exact diagonalization of a many-body Hamiltonian. The many-body conductivity reveals a Brinkman-Rice metal-insulator transition at a critical interaction strength; the calculated interaction strength is well above this critical value.
\end{abstract}

73.20.-r, 71.30.+h, 71.10.Fd, 71.27.+a

Transport behavior in crystalline materials is governed by the excitation spectrum: insulators have a finite gap to excitations while metals have zero-energy excitations. Band theory accurately describes this distinction in most materials: systems with only filled or empty bands are insulating while systems with partially occupied bands are metallic. However, the band description may break down under circumstances when, roughly speaking, the energy cost for forming an extended state exceeds the cost for forming a localized state. The resulting ground state, which arises from electron-electron interactions that band theory cannot describe, is known as a Mott insulator [1] [3].

Surfaces provide a potentially fertile environment for Mott insulators [- Electrons occupying surface states may localize more readily than in the bulk, due to two significant effects: (1) Atoms at surfaces have lower coordination than in the bulk, raising the energetic cost for electron hopping. (2) Surfaces often undergo reconstructions, yielding much larger inter-orbital spacings than in the bulk. These effects combine to make surfaces natural systems to look for Mott insulating behavior. In a recent series of experiments, Weitering et al. [5, 6] used photoemission and inverse photoemission to demonstrate that the $\mathrm{K} / \mathrm{Si}(111)-(\sqrt{3} \times \sqrt{3})-\mathrm{B}$ surface has a gap at the Fermi level. Since this system has an odd number of electrons per unit cell it must be metallic in a band description, clearly contradicting the photoemission data. On this basis, Weitering et al. hypothesized that this system (hereafter $\mathrm{K} / \mathrm{Si}-\mathrm{B}$ ) is a Mott insulator.

In this Letter we explicitly demonstrate, by exact solution of the appropriate many-body Hamiltonian, that the electronic ground state of $\mathrm{K} / \mathrm{Si}-\mathrm{B}$ is indeed a Mott insulator. The calculation is in three parts. First, we use standard density-functional methods to determine the geometrical and electronic structure of this surface within the local-density approximation (LDA). Second, we map the relevant electronic states onto a many-body Hamiltonian, which we then solve on a periodic cluster using exact diagonalization techniques. Third, we use the resulting many-body ground state to compute the zero-

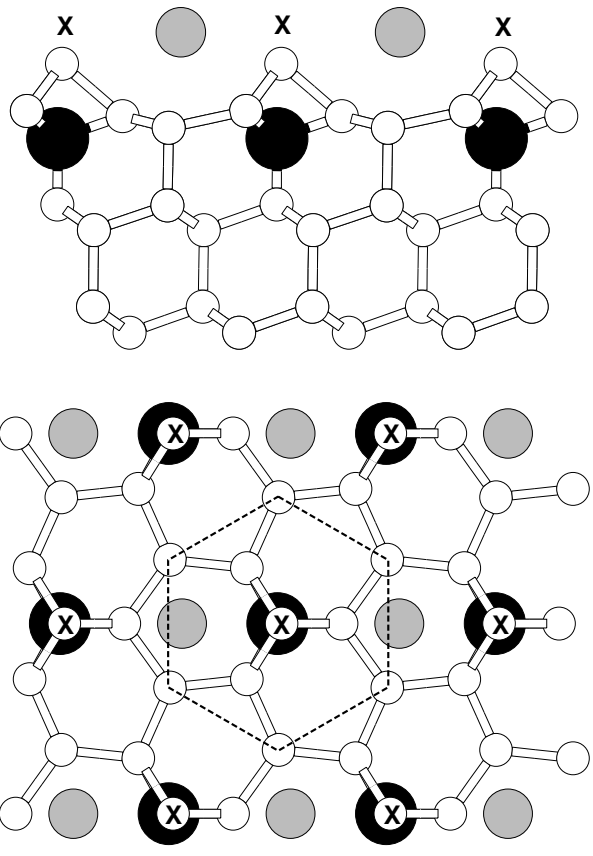

FIG. 1. Side and top views of the fully relaxed structure of $\mathrm{K} / \mathrm{Si}(111)-(\sqrt{3} \times \sqrt{3})$ - $\mathrm{B}$. K atoms are gray, Si atoms are white, and B atoms are black. The hexagon outlines the unit cell, and X's denote Si dangling-bond orbitals.

frequency conductivity or Drude weight, $D$, and then show that in the infinite system $D \rightarrow 0$, that is, a metalinsulator transition occurs in the thermodynamic limit.

Boron induces a well-known $\sqrt{3} \times \sqrt{3}$ reconstruction of the clean $\operatorname{Si}(111)$ surface [7]. Boron substitutes for every third Si atom in the second subsurface layer, and the displaced $\mathrm{Si}$ assumes an adatom position above the boron (see Fig. 1). The electron in the Si-adatom dangling bond is transferred subsurface, enabling the B atom to participate in four covalent bonds. By this mechanism, the surface forms a conventional band insulator, leaving each $\mathrm{Si}$ adatom with an empty orbital extending away from the surface. These orbitals form a triangular lattice on the surface. In the experiments of Weitering et al., $\mathrm{K}$ was then deposited onto this insulating substrate until 


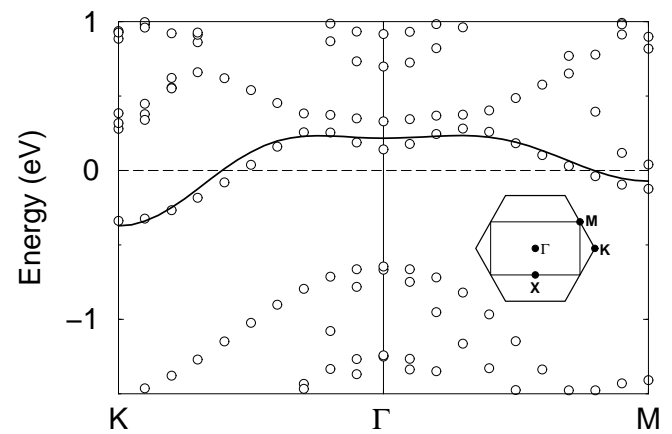

FIG. 2. LDA band structure of $\mathrm{K} / \mathrm{Si}-\mathrm{B}$ at the $\mathrm{K}$ saturation coverage of $1 / 3 \mathrm{ML}$. The Si-related band crossing the Fermi level is half occupied by the $\mathrm{K} 4 s$ electron. The solid curve is the optimized fit of the mean-field Hubbard dispersion, Eq. (2), to the LDA eigenvalues. The inset shows the Brillouin zone of the fundamental unit cell and of the doubled supercell, used to compute $U_{\mathrm{K} / \mathrm{Si}-\mathrm{B}}$.

the saturation coverage was reached.

To determine the equilibrium structure of $\mathrm{K} / \mathrm{Si}-\mathrm{B}$, we have performed extensive LDA calculations. The calculations used a slab geometry with three double layers of $\mathrm{Si}$, terminated by $\mathrm{H}$, and a vacuum region equivalent to three double layers of Si. Total energies and forces were calculated using Hamann and Troullier-Martins pseudopotentials, and a plane-wave basis with a kinetic-energy cutoff of $20 \mathrm{Ry}$, as implemented in the FHI96mD code [8]. Four k-points were used for Brillouin-zone integrations. Full structural relaxation was performed on all atoms, except those in the bottommost double layer, until the rms force was less than $0.05 \mathrm{eV} / \AA$. We began by first fully relaxing the surface without $\mathrm{K}$ present, and then proceeded to determine the equilibrium coverage and geometry of the K-saturated surface.

Experimentally, coverage is monitored via the electron work function: at the saturation coverage, the lowtemperature work function reaches a minimum [6]. The absolute $\mathrm{K}$ coverage is not known from experiment, so it must be determined theoretically. We calculated the work function for the lowest-energy arrangement of adsorbates at coverages of $1 / 6,1 / 3,2 / 3$, and 1 monolayer (ML), and find a minimum at $1 / 3 \mathrm{ML}$, in agreement with the conclusions of Weitering et al. At all coverages, the experimental photoemission spectra show that the $\mathrm{Si}$ adatom backbond state persists upon $\mathrm{K}$ deposition, suggesting the $\mathrm{K}$ adsorbates do not break the $\mathrm{Si}$ adatom bonds [6]. We therefore assume that at these coverages the adsorbates do not destroy the underlying reconstruction. The resulting minimum energy configuration at the saturation coverage of $1 / 3 \mathrm{ML}$ is shown in Fig. 1. The $\mathrm{K}$ adsorbates are in the $H_{3}$ hollow site, with the $\mathrm{Si}$ adatom slightly shifted from its position with no $\mathrm{K}$ present. A metastable state with the $\mathrm{K}$ adsorbate in the $T_{4}$ hollow site has an energy $0.1 \mathrm{eV}$ higher per unit cell.
At coverages below $2 / 3 \mathrm{ML}$, one expects the $\mathrm{K} 4 s$ electrons to partially occupy the surface state arising from the empty Si-adatom orbitals. At $1 / 3 \mathrm{ML}$ there is one $\mathrm{K}$ per Si orbital, so in the band description the single surface band is half occupied; this simple picture is confirmed by the calculated LDA band structure shown in Fig. 2. Clearly this system must be metallic within band theory. To investigate the importance of electronic interactions not included in band theory, we derive a singleband Hubbard model for the half-filled surface state. The Hamiltonian is

$$
H=\sum_{i j \sigma} t_{i j} c_{i \sigma}^{\dagger} c_{j \sigma}+U \sum_{i} n_{i \uparrow} n_{i \downarrow}
$$

where $c_{i \sigma}^{\dagger}$ creates an electron with spin $\sigma$ on site $i$, and $n_{i \sigma}=c_{i \sigma}^{\dagger} c_{i \sigma}$ is the number operator. The sites correspond to the empty $\mathrm{Si}$ orbitals that the K electrons are doping. The amplitude for hopping from orbital $i$ to orbital $j$ is given by $t_{i j}$, and $t_{i j}=t_{j i}$. There is a Coulomb energy cost of $U$ to occupy an orbital with two electrons.

Our approach to determining the parameters of the Hubbard model is similar to other first-principles approaches [9, 10]. We first solve the Hubbard model in the mean-field (MF) approximation, and then require that the resulting single-particle energies optimally reproduce the corresponding LDA spectrum (which is also a meanfield theory) throughout the zone. In the MF approximation, the up electrons move in the average potential generated by the down electrons (and vice-versa), so the MF Hubbard Hamiltonian for the up electrons becomes

$$
H_{\uparrow}^{\mathrm{MF}}=\sum_{i j} t_{i j} c_{i \uparrow}^{\dagger} c_{j \uparrow}+U \sum_{i} n_{i \uparrow}\left\langle n_{i \downarrow}\right\rangle,
$$

where $\left\langle n_{i \downarrow}\right\rangle$ is the average density of the down electrons on site $i$. We assume a paramagnetic state in both the LDA and the MF solution to the Hubbard model.

To determine the hopping amplitudes, $t_{i j}$, we fit the single-particle eigenvalues in the MF Hubbard solution to the LDA eigenvalues of the surface band at 100 special $k$-points. Note that although the $\mathrm{K}$ adsorbates break the three-fold rotational symmetry of the substrate, the LDA band structure remains nearly isotropic, and so we assume isotropic hopping. We allow hopping between nearest-neighboring and secondnearest-neighboring sites, with amplitudes $t_{1}$ and $t_{2}$ respectively - Third-nearest-neighbor hopping was found to be insignificant. Thus the dispersion is given by

$$
\begin{aligned}
\varepsilon(\mathbf{k}) & =2 t_{1}\left[\cos \left(k_{y}\right)+2 \cos \left(k_{x} \sqrt{3} / 2\right) \cos \left(k_{y} / 2\right)\right] \\
& +2 t_{2}\left[\cos \left(k_{x} \sqrt{3}\right)+2 \cos \left(k_{x} \sqrt{3} / 2\right) \cos \left(3 k_{y} / 2\right)\right] .
\end{aligned}
$$

The optimized amplitudes are $t_{1}=66 \mathrm{meV}$ and $t_{2}=-24$ $\mathrm{meV}$. A plot of the fit and the LDA eigenvalues along 


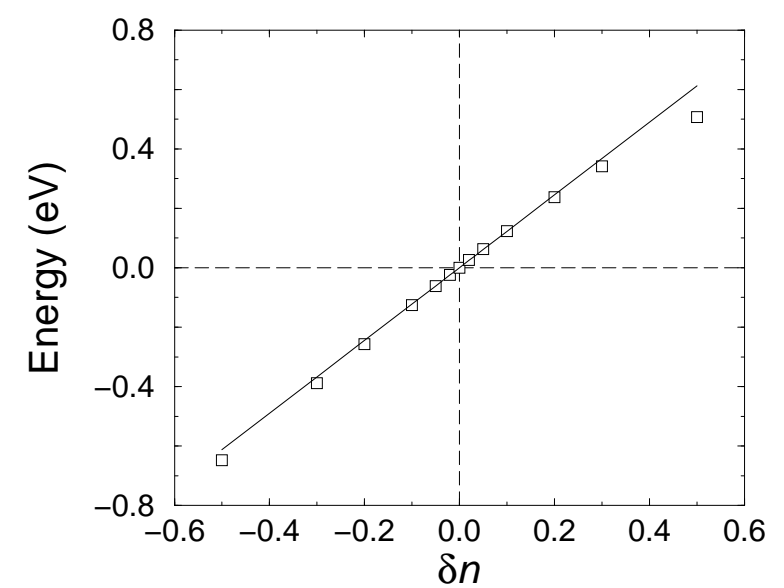

FIG. 3. The single-particle energy $\varepsilon_{+}$at the edge of the Brillouin zone (the $\mathrm{X}$ point), as a function of the electron transfer $\delta n$. The squares are LDA supercell results; the solid line is the best fit of Eq. (何) to the LDA results for $|\delta n| \leq 0.3$.

high symmetry directions is shown in Fig. 2; the fit is very good, with a rms error of $42 \mathrm{meV}$.

To determine the intra-orbital Coulomb repulsion $U$, we subject the system to a density fluctuation by moving charge from one Si orbital to another. The optimal interaction parameter, $U_{\mathrm{K} / \mathrm{Si}-\mathrm{B}}$, is then determined by requiring the LDA solution and the MF Hubbard calculation to respond identically. In order to maintain overall charge neutrality, a supercell calculation with two Si orbitals is required. At the edge of the Brillouin zone, the paramagnetic single-particle eigenvalues for a shift of $\delta n$ of an electron from one orbital to another take the simple form

$$
\varepsilon_{ \pm}= \pm U \delta n / 2
$$

up to an overall constant which we take to be zero. The fit of $\varepsilon_{+}$to the LDA eigenvalues is shown in Fig. 3. For small charge shifts, the LDA eigenvalues are nearly linear, and we obtain $U_{\mathrm{K} / \mathrm{Si}-\mathrm{B}}=1.23 \mathrm{eV}$. For larger charge shifts, additional bands in the LDA calculation enter that are not present in the single-band Hubbard model, and the LDA eigenvalues drop very slightly below the MF Hubbard eigenvalues. As a check of the reliability of this approach, we also determined $U_{\mathrm{K} / \mathrm{Si}-\mathrm{B}}$ by fitting the change in the total kinetic energy in the MF Hubbard solution to the LDA. We find $U_{\mathrm{K} / \mathrm{Si}-\mathrm{B}} \approx 1.2 \mathrm{eV}$, consistent with the above result.

Having determined the parameters of the Hubbard model describing $\mathrm{K} / \mathrm{Si}-\mathrm{B}$, we now solve this model exactly (using a periodic 16-site cluster) to obtain the many-body electronic ground state. The number of states in the Hilbert space grows exponentially with the number of sites in the cluster: in the Hubbard model, each site can have zero, one (either up or down), or two electrons, so the number of basis states in the space of an $N$-site system is $4^{N}$. The symmetries of the Hamiltonian make the matrix block diagonal, but with 16 sites the size of the largest block is still more than $10^{7} \times 10^{7}$. Conventional algorithms obviously cannot diagonalize matrices this large, and so we use the Lanczos algorithm to determine the exact ground state [11]. Storing the Hamiltonian and three basis vectors in memory on an IBM SP2 required 64 nodes with $1 \mathrm{~Gb}$ memory each; the computation required about $600 \mathrm{CPU}$ hours.

To distinguish quantitatively between metallic and insulating behavior, we calculate the zero-frequency conductivity or Drude weight, $D$, of the many-body ground state. The Drude weight provides a definitive way to distinguish metals from insulators irrespective of the applicability of band theory: in the thermodynamic limit, metals have non-zero Drude weight, while for insulators $D=0$. Kohn first showed that $D$ may be calculated from the variation of the ground-state energy with respect to an applied vector potential [2, 3, 12],

$$
D=\partial^{2} E / \partial \phi^{2} \text {. }
$$

We use this technique to determine the Drude weight of our Hubbard cluster as a function of the interaction parameter $U$. The results are shown in Fig. 4 . At small $U$, the system is in the metallic regime and so $D$ is large, and it decreases monotonically with increasing $U$. $D$ never becomes zero, because only an infinite system can undergo a true metal-insulator transition. Instead of a critical interaction strength, our 16-site cluster shows a transition region in the vicinity of the physically interesting interaction strengths, near $U=1 \mathrm{eV}$.

In general, there may be level crossings in the ground state as a function of $U$. For our hopping amplitudes, this is not the case - The ground state evolves adiabatically with increasing $U$, and the Drude weight in Fig. 1 is a continuous function. We see no evidence of the intermediate (semi-metallic) phase that was found in recent slave-boson studies of the Hubbard model on a triangular lattice with $t_{2}=0$ [13].

To show that K/Si-B lies on the insulating side of a Mott-insulator transition, we need the critical interaction strength for the transition in order to compare with our calculated interaction strength, $U_{\mathrm{K} / \mathrm{Si}-\mathrm{B}}$. We can obtain this value by extending our calculated $D(U)$ from the exact finite-cluster result to the thermodynamic infinite limit, using the form derived with the Gutzwiller approximation [1] for the infinite system,

$$
D_{\infty}(U) \propto \begin{cases}1-\left(U / U_{c}\right)^{2}, & U<U_{c} \\ 0, & U>U_{c}\end{cases}
$$

where $U_{c}$ is the critical interaction strength for the metalinsulator transition. This functional form fits our 16-site results at small and intermediate values of $U$ extremely well, as shown in Fig. 1. From this fit, we estimate the critical interaction strength for the Mott metal-insulator transition in the infinite system occurs at $U_{c}=0.95 \pm$ $0.02 \mathrm{eV}$. Our calculated value for the physical system, 


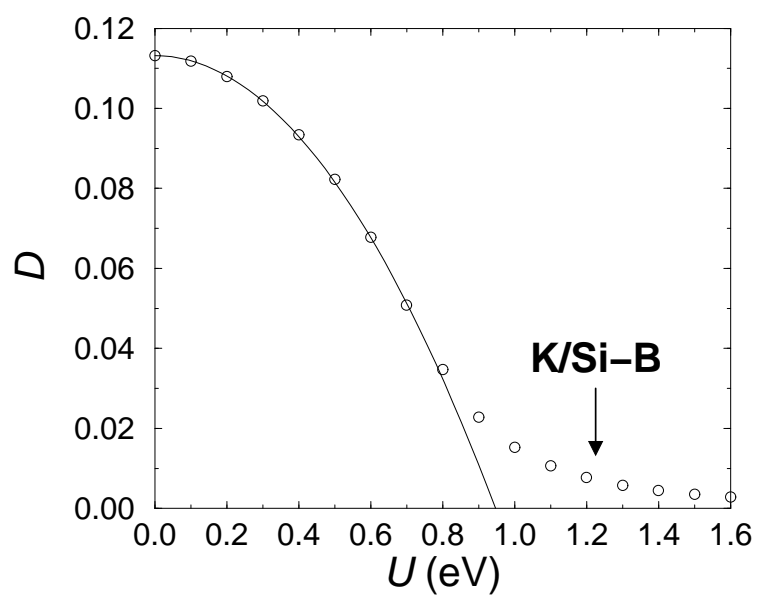

FIG. 4. The Drude weight, $D$, as a function of the interaction parameter $U$. The circles are exact results from the Hubbard model on a 16-site cluster, and the curve is a fit of the Hubbard results (for $U \leq 0.7 \mathrm{eV}$ ) to the infinite limit given by Eq. (6).

$U_{\mathrm{K} / \mathrm{Si}-\mathrm{B}}=1.23 \mathrm{eV}$, is well above this critical value, and establishes that $\mathrm{K} / \mathrm{Si}-\mathrm{B}$ is indeed a Mott insulator.

In the Mott-insulating regime, the localized electrons will interact via an antiferromagnetic Heisenberg exchange coupling with strength $J_{i j}=4 t_{i j}^{2} / U$ [14]. The Heisenberg model on a triangular lattice is frustrated. The nearest-neighbor model has strong three-sublattice correlations, but whether the correlations are longranged is controversial [15]. In the Hubbard model for $\mathrm{K} / \mathrm{Si}-\mathrm{B}$, the second-neighbor hopping is substantial, so the second-neighbor antiferromagnetic Heisenberg coupling in the Mott-insulating limit will be significant. This coupling frustrates the three-sublattice correlations in the nearest-neighbor model, so it is unlikely that threesublattice order is established. The ground state will likely either establish some other type of collinear order [16] or enter a quantum-disordered regime with no longrange order [2].

To conclude, we have shown that the many-body electronic ground state of the K/Si-B surface is a Mott insulator. Specifically, we have first determined the surface coverage and morphology of $\mathrm{K}$ adsorbed on $\mathrm{Si}(111)$ $\mathrm{B}$ using first-principles total-energy methods. We then mapped the relevant electronic degrees of freedom onto a Hubbard model, which we solved with exact diagonalization. By calculating the Drude weight of the model, we have demonstrated that for the physical parameters of $\mathrm{K} / \mathrm{Si}-\mathrm{B}$, the model has a Mott insulating ground state.

We thank H. H. Weitering, E. J. Mele, M. J. Rozenberg, and D. W. Hess for enlightening discussions. This work was supported by the National Research Council and was funded by ONR. Computational work was supported by a grant of HPC time from the DoD Major Shared Resource Center ASCWP.
* Electronic address: hellberg@dave.nrl.navy.mil.

$\dagger$ On leave at: Fritz-Haber-Institut, Faradayweg 4-6, D14195 Berlin-Dahlem, Germany.

[1] W. F. Brinkman and T. M. Rice, Phys. Rev. B 2, 4302 (1970); X. Y. Zhang, M. J. Rozenberg, and G. Kotliar, Phys. Rev. Lett. 70, 1666 (1993); A. Georges, G. Kotliar, W. Krauth, and M. J. Rozenberg, Rev. Mod. Phys. 68, 13 (1996).

[2] F. Gebhard, The Mott Metal-Insulator Transition (Springer, New York, 1997).

[3] M. Imada, A. Fujimori, and Y. Tokura, Rev. Mod. Phys. 70, 1039 (1998).

[4] J. M. Carpinelli, H. N. Weitering, E. W. Plummer, and R. Stumpf, Nature 381, 398 (1996).

[5] H. H. Weitering et al., Phys. Rev. Lett. 78, 1331 (1997).

[6] H. H. Weitering, J. Chen, N. J. DiNardo, and E. W. Plummer, Phys. Rev. B 48, 8119 (1993).

[7] I.-W. Lyo, E. Kaxiras, and Ph. Avouris, Phys. Rev. Lett. 63, 1261 (1989); H. Huang, S. Y. Tong, J. Quinn, and F. Jona, Phys. Rev. B 41, 3276 (1990); E. Kaxiras, K. C. Pandey, F. J. Himpsel, and R. M. Tromp, ibid. 41, 1262 (1990); S. Wang, M. W. Radny, and P. V. Smith, J. Phys. Cond. Mat. 9, 4535 (1997).

[8] M. Bockstedte, A. Kley, J. Neugebauer, and M. Scheffler, Comp. Phys. Comm. 107, 187 (1997).

[9] O. Pankratov and M. Scheffler, Phys. Rev. Lett. 70, 351 (1993); X. W. Wang and C. Chen, Phys. Rev. B 54, 13436 (1996).

[10] M. S. Hybertsen, M. Schlüter, and N. E. Christensen, Phys. Rev. B 39, 9028 (1989); A. K. McMahan, J. F. Annett, and R. M. Martin, ibid. 42, 6268 (1990); S. B. Bacci, E. R. Gagliano, R. M. Martin, and J. F. Annett, ibid. 44, 7504 (1991); R. M. Martin, Int. J. Mod. Phys. C 2, 105 (1991).

[11] J. K. Cullum and R. A. Willoughby, Lanczos Algorithms for Large Symmetric Eigenvalue Computations (Birkhauser, Boston, 1985).

[12] W. Kohn, Phys. Rev. 133, A171 (1964); B. S. Shastry and B. Sutherland, Phys. Rev. Lett. 65, 243 (1990); D. J. Scalapino, S. R. White, and S. Zhang, Phys. Rev. B 47, 7995 (1993).

[13] C. J. Gazza, A. E. Trumper, and H. A. Ceccatto, J. Phys. Cond. Mat. 6, L625 (1994).

[14] A. Auerbach, Interacting Electrons and Quantum Magnetism (Springer, New York, 1994).

[15] D. A. Huse and V. Elser, Phys. Rev. Lett. 60, 2531 (1988); P. W. Leung and K. J. Runge, Phys. Rev. B 47, 5861 (1993); B. Bernu, P. Lecheminant, C. Lhuillier, and L. Pierre, ibid. 50, 10048 (1994); M. Boninsegni, ibid. 52, 15304 (1995).

[16] Th. Jolicoeur, E. Dagotto, E. Gagliano, and S. Bacci, Phys. Rev. B 42, 4800 (1990); P. Lecheminant, B. Bernu, C. Lhuillier, and L. Pierre, ibid. 52, 6647 (1995). 